\title{
Fabrication of three-dimensional structures for the assessment of cell mechanical interactions within cell monolayers
}

\author{
David Fuard, Michel Moussus, Caterina Tomba, David Peyrade, and Alice Nicolas ${ }^{a)}$ \\ Laboratoire des Technologies de la Microelectronique, CNRS-UJF-Grenoble INP, 17 av des Martyrs, 38054 \\ Grenoble Cedex, France
}

(Received 15 July 2010; accepted 4 October 2010; published 10 November 2010)

\begin{abstract}
The measurement of the forces at play in cell/cell adhesion uses a cell monolayer plated onto two-dimensional substrate like arrays of elastomeric microfabricated pillars. Unfortunately, the few attempts that have been done in this direction do not mimic the extracellular environment and lack knowledge on the correlation between the various types of cellular adhesions. Here, the authors suggest using a new experimental setup that more accurately simulates the three-dimensional (3D) environment of cells in tissues, using stretchable hexagonal monocellular 3D structures. The present article shows a way of fabricating these hexagonal biosensors, which are open structures made of biocompatible elastomeric polydimethylsiloxane (PDMS). The novelty of the whole fabrication process of these 3D PDMS structures consists in the use of a sacrificial silicon mold. An original mechanical PDMS planarization process is proposed. This article also describes a strategy for a selective functionalization of the 3D structure sidewalls where the cells must adhere. (0) 2010 American Vacuum Society. [DOI: 10.1116/1.3511435]
\end{abstract}

\section{INTRODUCTION}

The organization of biological cells is sensitive not only to the biochemical composition of their environment but also to its mechanical properties. For instance, the physical/ chemical nature of the external medium and its binding with the transmembrane proteins responsible for adhesion allow cells to probe the elastic properties of their environment. ${ }^{1,2}$ Consequently, cells are able to discriminate patterns of rigidity of a surface on which they are placed and to migrate to the more rigid part. ${ }^{3-5}$ The sensitivity of cells to the mechanical properties of the extracellular matrix arises from the mechanosensitive nature of cell adhesion. ${ }^{6-8}$ Recent studies show that adhering cells actively probe the physical properties of the extracellular matrix by pulling onto it through its adhesive regions ${ }^{9-11}$ and respond by modulating their adhesion or their migration activity. ${ }^{9} 12-14$ A key issue now is to elucidate the stresses that cells transmit to each other in tissues. Measuring the forces at play in cell/cell adhesion is challenging, since the use of a force sensor in a cell monolayer leads to the emergence of cell/extracellular matrix adhesions. Few attempts have been done in this direction ${ }^{15,16}$ using cell monolayer or cell doublet ${ }^{17}$ plated onto twodimensional (2D) substrate like arrays of elastomeric microfabricated pillars, but their analysis lacks knowledge on the correlation between the two types of adhesions. Recently, isolated cells were plated onto 2D substrates with microfabricated pillars coated with cell/cell adhesion proteins ${ }^{18,19}$ or with pillars able to stress the cell as would do neighboring cells. ${ }^{20}$ However, cell geometry is known to influence gene expression $^{21}$ and many cellular processes such as cell division $^{22}$ or cell adhesion. ${ }^{23}$

\footnotetext{
a) Author to whom correspondence should be addressed; electronic mail: alice.nicolas@cea.fr
}

In order to face the aforementioned limitations when estimating intercellular mechanical interactions, we suggest using a new experimental setup that simulates more accurately the three-dimensional (3D) environment of cells in tissues using stretchable hexagonal monocellular 3D structures, both connected in series and in parallel distributions (see Fig. 1). Every cell in a hexagonal deformable well is constrained in a shape that resembles the shapes of cells in tissues in an embryo $^{24}$ and are close to the average cell shape in in vitro cell monolayers. An external mechanical device is to be fixed to the microfabricated sensor, which imitates forces exerted by neighboring cells. Our future goal is to measure the dynamics of the cellular response to such external stretching. In the presence of a cell in the hexagonal wells, we expect that the observed deformation (for instance, the variation of the angle $\theta$ in Fig. 1) is smaller in the presence of a cell than for a stretched, empty well. An analysis of the deformation of the hexagons will give us information on both the passive contribution of the cell to the deformation of the sensor (elasticity and viscosity) and on the active contribution of its contractile, cytoskeletal machinery. ${ }^{25}$ Due to their material properties, the magnitude of the forces that cells apply should be on the order of $\mathrm{nN} / \mu \mathrm{m}^{2}{ }^{26}$ This is indeed what is observed in the well studied case of cell adhesion onto an extracellular polymeric matrix. ${ }^{9,10}$ Such order of magnitude for the forces exchanged between cells is therefore to be expected. For this reason, the force sensor we propose does not aim at giving a precise quantification of the intercellular forces but at measuring the dynamics of the mechanical response of the cells to mechanical stimuli such as the ones they are exposed to in a tissue. In the present article, we focus on the geometrical design and the microfabrication of the device, including the chemical functionalization of the surfaces that will enable the cells to localize into the hexagonal structures. Complex 3D patterning strategies have been 

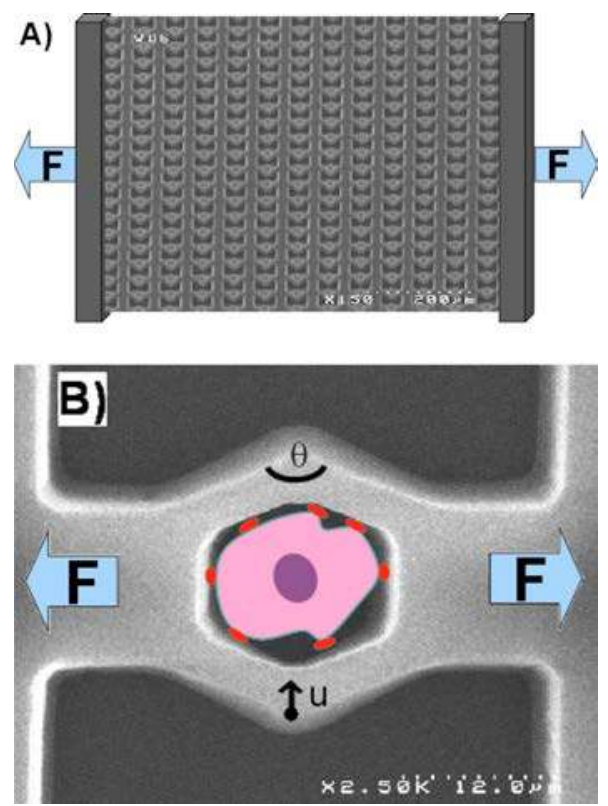

FIG. 1. (Color online) Force sensor for the measure of the mechanics of intercellular forces. Each hexagon will contain a unique cell. An externally applied force $F$ imitates the forces from neighboring cells that cells meet in cellular monolayers. (A) The overall sensor consists of parallelized and series hexagons. (B) Focus on one hexagonal structure. The measure of the variation of the angle $\theta$ or equivalently of the displacement $u$ of the labeled point perpendicular to the force $F$ gives information on the viscoelastic behavior of both the hexagonal frame and the cell inside it.

done before using hard materials such as resist, ${ }^{27,28}$ but these strategies do not meet our requirements for the use of deformable biocompatible materials such as polydimethylsiloxane (PDMS). In addition, we aim at making open PDMS structures, so that the cells do not build basal adhesions and keep a geometry that resembles their organization in tissues. For all these reasons, the strategy we describe here uses a sacrificial silicon mold followed by an original planarization process that enables the opening of the PDMS structures. We first describe the processes used for this purpose. In a second part, we report on the efficiency of the processes we developed, and we eventually discuss the limitations of the proposed strategy.

\section{EXPERIMENTAL SETUP AND METHODS}

The hexagonal structure fabrication is carried out by means of several commercial technological tools, which are commonly used for making microelectromechanical systems or integrated circuits. The experimental details below follow the sequence of the technical processes used for the sensor fabrication (see Fig. 3).

\section{A. Design of the silicon mold}

The silicon mold designs are printed on pieces of silicon wafer using standard lithography steps: the pieces of silicon are first dried and turned hydrophilic via oxygen plasma for a better adhesion of the promoter and the photoresist. This plasma is generated using a Plassys MDS 150A tool with a $25 \mathrm{~cm}^{3} / \mathrm{min}$ (SCCM, where SCCM denotes cubic centime- ter per minute at STP) oxygen flow and a $500 \mathrm{~W}$ source power for $30 \mathrm{~s}$. The TI PRIME adhesion promoter (MicroChemicals) is then spun on the silicon substrates at $3000 \mathrm{rpm}$ for $30 \mathrm{~s}$ using the standard two-step speed up process. This spin on adhesion promoter is hotplate baked at $120^{\circ} \mathrm{C}$ for 2 min. A positive AZ 1512HS photoresist (MicroChemicals) is then spun on this promoter. The pattern for the etching mask definition is achieved by a contact lithography process using a manual UV400 MJB4 mask aligner from Süss Microtech $(200 \mathrm{~W})$. The mask pattern is transferred with $72^{\circ}$ sidewall angle into the photoresist due to diffraction effects at the mask pattern edges, which leads to a $0.7 \mu \mathrm{m}$ pattern shrinking of the features' bottom for a $2 \mu \mathrm{m}$ thick photoresist [Fig. 3(a)]. Pattern shrinking was reduced by limiting the thickness of the resist. The AZ 1512 HS photoresist was mixed with its solvent (propylene glycol monomethyl ether acetate or AZ EBR solvent) in the proportion 1:1. We obtained a $0.6 \mu \mathrm{m}$ thick layer of AZ 1512 photoresist with a $3000 \mathrm{rpm}$ spin on process for $30 \mathrm{~s}$, leading to a pattern shrinking of $0.2 \mu \mathrm{m}$. The final resist pattern is developed with 1:1 AZdeveloper:de-ionized water mixture for $1 \mathrm{~min}$ [Fig. 3(b)].

The photoresist mask is transferred into the silicon by plasma deep reactive ion etching using the Bosch process ${ }^{29}$ with a surface technology system (STS) etcher, where both the source antenna and the bottom electrode are powered [Fig. 3(c)]. Twenty cycles of etching and deposition are performed. The etching parameters are set to $2000 \mathrm{~W}$ plasma source power, $50 \mathrm{~W}$ bias power, $60 \mathrm{mTorr}$ pressure, and 400 SCCM SF6/20 SCCM C4F8/30 SCCM O 2 gas mixture flow with an etching time increasing from $3 \mathrm{~s}$ at the beginning of the process to $4 \mathrm{~s}$ for the last etching cycle. The deposition cycle parameters are set to $2 \mathrm{~s}$ duration, $2000 \mathrm{~W}$ source power, no bias power, 15 mTorr pressure, and 200 SCCM C4F8 flow.

\section{B. Fabrication and unmolding of the PDMS structures}

A biocompatible bicomponent elastomeric PDMS SYLGARD 184, from Dow Corning, is used for the structure production. The two PDMS components are thoroughly mixed using a 10:1 base to curing agent mixing ratio in mass and degassed under vacuum $\left(10^{-4} \mathrm{~atm}\right)$ to remove the bubbles produced during the mixing. The mixture is eventually annealed at $100{ }^{\circ} \mathrm{C}$ for $15 \mathrm{~min}$ to obtain a $1 \mathrm{MPa}$ elastic modulus polymer $^{30}$ (see Sec. III A) [Fig. 3(d)]. Unmolding of the PDMS device will be performed by selectively etching the silicon mold, thus preventing unmolding-induced mechanical deformation of the PDMS structure. To this aim, the residual PDMS layer on top of the mold must be etched. Preliminary planarization of the PDMS is necessary, so that the final PDMS microstructures have a homogeneous thickness through the whole device. Planarization proceeds before thermal reticulation by pressing a flat silicon master supporting a polyethylene film coated with AZ 1512 HS resist film [Fig. 3(d)]. The resist is deposited onto the polyethylene film using the same lithographic recipe as initial silicon wafer samples used for mold fabrication: an oxygen plasma in Plassys MDS 150 A turns the polyethylene surface to hydro- 
philic and the resist spin on is possible after TI PRIME adhesion promoter spin on. This polyethylene film coated with AZ 1512 HS resist is then placed between PDMS and a flat piece $\left(25 \times 6 \mathrm{~mm}^{2}\right)$ of silicon wafer. A squeezing force of 50 $\mathrm{N}$ is applied for at least $1 \mathrm{~min}$ on the silicon master.

After thermal reticulation, the polyethylene film is peeled off the PDMS, and the PDMS residual layer is removed in an inductively coupled industrial plasma source [decoupled plasma source (DPS) from Applied Materials, Inc.]. Details of the DPS source may be found elsewhere. ${ }^{31,32}$ The PDMS is etched with a $1: 2 \mathrm{O}_{2}: \mathrm{CF}_{4}$ gas mixture, close to the 1:3 gas mixture usually applied in other works, ${ }^{33,34}$ with $1000 \mathrm{~W}$ source power, $100 \mathrm{~W}$ bias power, and $10 \mathrm{mTorr}$ gas pressure [Fig. 3(f)]. This provides a uniform PDMS etching rate of $1 \mu \mathrm{m} / \mathrm{min}$ with quasi-infinite selectivity with the silicon mold.

Unmolding of the PDMS device is conducted by selectively etching the silicon mold using the same Bosch process recipe as for mold etching [Fig. $3(\mathrm{~g})]$. The etching selectivity of Si:PDMS is $50: 1$.

\section{Selective 3D functionalization of the device}

AZ 1512 HS photoresist is used as a mask to limit the adsorption of the adhesion proteins to the regions of the PDMS device where the cells must localize (see Fig. 5).

An oxygen plasma treatment (Plassys MDS $150 \mathrm{~A}$ ) is applied for $30 \mathrm{~s}$ to make the polymer surface hydrophilic. The water drop contact angle decreases from $110^{\circ}$ before oxidation to a value below $10^{\circ}$ (PDMS in air recovers hydrophobicity on the scale of $1 \mathrm{~h}) .{ }^{35} \mathrm{AZ} 1512 \mathrm{HS}$ photoresist is spun on the surface $(100 \mathrm{rpm} / \mathrm{s}, 1500 \mathrm{rpm}$, and $30 \mathrm{~s})$ and baked to evaporate the solvent $\left(100{ }^{\circ} \mathrm{C}, 3 \mathrm{~min}\right)$. Low acceleration ensures that the $10 \mu \mathrm{m}$ deep structures are filled with the resist. The substrate is aligned with a chromium mask that hides regions that have to be kept protected from surface treatment by the resist. Exposition time to UV is $19 \mathrm{~s}$ $\left(8 \mathrm{~mW} / \mathrm{cm}^{2}\right)$ in soft contact mode. After development in AZ developer, the surface is turned hydrophilic by exposure to oxygen plasma. The substrate is then incubated in a 1:1 mixture of fibronectin/Alexa 488 labeled fibrinogen at $20 \mu \mathrm{g} / \mathrm{ml}$ (Invitrogen) for $1 \mathrm{~h}$. The resist is rinsed in ethanol for $50 \mathrm{~s}$ and sonicated for an additional $30 \mathrm{~s}$. The sensor is then immersed in a 1\% solution of Pluronic F127 (Sigma).

\section{RESULTS}

\section{A. Design of the sensor}

The average size of animal cells is on the order of $10 \mu \mathrm{m}$ when they are suspended in a solution. Depending on cell type, they may spread on a substrate and extend up to about $50 \mu \mathrm{m}$. Cells in monolayers are less spread. They exhibit a columnar shape and their height is largely dependent on the interaction they have with the extracellular layer underneath. For our purpose, we want to uncouple the response of intercellular adhesions from cell/matrix adhesions. We therefore design a force sensor with no basal matrix. Cells will only have the opportunity to attach to the lateral sides of the hex-

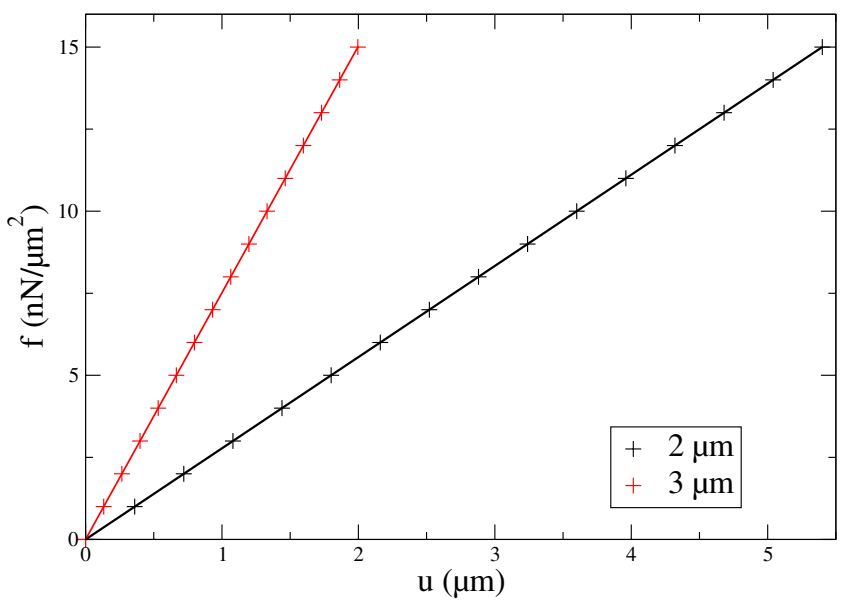

FIG. 2. (Color online) Finite element calculation of the displacement $u$ with the stress $f$ for two thicknesses of the frame of the hexagons. $f$ is the force $F$ per unit of the surface that is pulled (see Fig. 1 for the notations).

agonal wells that are to be functionalized with a protein that is involved in intercellular adhesion (for instance, from the cadherin family). Each hexagonal well of the force sensor should host a unique cell (see Fig. 1). We therefore choose wells with dimensions as represented in Fig. 1. The wells are designed with a depth of $10 \mu \mathrm{m}$. The depth will be optimized depending on the cell type.

The thickness of the hexagonal frame determines the elastic properties of the force sensor. As discussed in the Introduction, expected forces are on the order of $\mathrm{nN} / \mu \mathrm{m}^{2}$. The deformation of the hexagons will be quantified by visualizing the displacement of the labeled point in Fig. 1 by phase contrast microscopy. Displacements must therefore be on the order of $1 \mu \mathrm{m}$ to ensure proper detection. Hexagons with a frame of thickness between 2 and $3 \mu \mathrm{m}$ and Young's modulus of $1 \mathrm{MPa}$ fulfill such conditions (see Fig. 2).

\section{B. Fabrication of the sensor}

The previously designed hexagonal features require the fabrication of a sacrificial mold for PDMS features' demolding. One way of hexagonal feature production may be the direct use of photosensitive PDMS, but the photosensitive PDMS has a weak resolution for feature sizes smaller than $10 \mu \mathrm{m}$ and has a poor pattern anisotropy. ${ }^{36,37}$ Another way could be to use a soluble sacrificial mold such as polyvinyl acetate (PVA), but this material is too soft to achieve a simple PDMS planarization process. In addition, PVA could not be placed under vacuum because of the presence of water in its composition. Since a reliable mold filling up by PDMS is achieved under vacuum and since PDMS planarization is conducted under etching plasmas, the use of PVA is difficult to apply here. Finally, among several approaches, the use of resist or silicon molds seems suitable for an easy sacrificial mold production. The hexagonal structure fabrication is based on several standard technological steps commonly available in clean room environments or open technological platforms. Besides, these processes are numerous and rather middle cost than low cost; they are easy to use without criti- 
a)

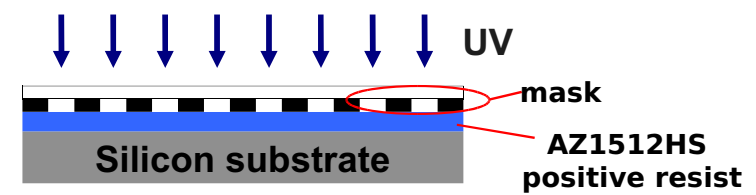

b) Silicon substrate

c)

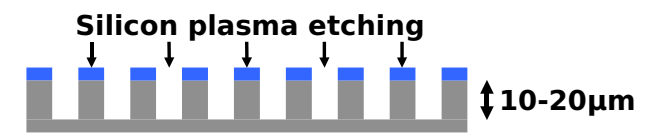

d)

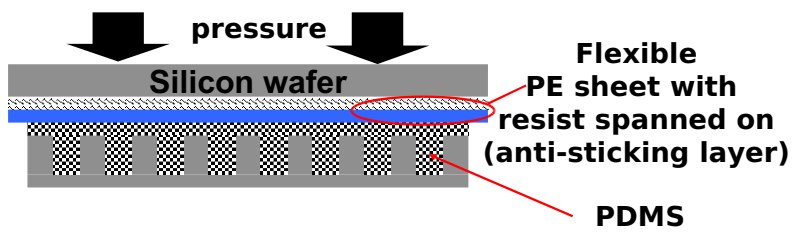

e)

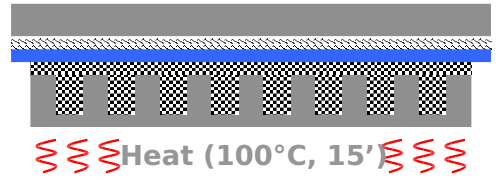

f)

$30 \% 02+70 \%$ CF4

High Density plasma etching
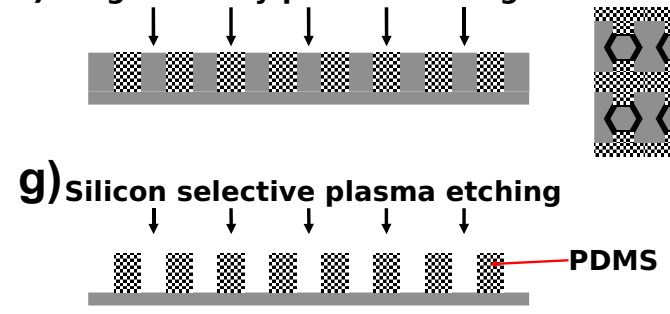

FIG. 3. (Color online) Schematic view of the hexagonal structure fabrication stages. (a) Lithography on silicon samples coated with AZ 1512 HS photoresist, (b) resist development, (c) deep reactive ion etching of the silicon mold, (d) silicon mold filling up with PDMS and PDMS planarization using a counter mold, (e) PDMS thermal cross-linking, and (f) opening of the top of the hexagonal structures by PDMS residual layer dry etching.

cal technical issues. Figure 3 shows the way of creating these hexagonal features using a silicon sacrificial mold that we described in this article. The broad lines of the process consist in casting PDMS into the mold, planarizing this PDMS onto the mold, removing this planarization layer to open the top of the hexagonal structures, and etching the sacrificial mold.

First, the standard lithographic process [Fig. 3(a)] described in Sec. II is carried out on silicon wafer pieces coming from cloven $200 \mathrm{~mm}$ silicon wafer. As the minimal width of the features is $2-3 \mu \mathrm{m}$, the resist thickness must be reduced to contain the pattern shrinking of the patterned features. Here, the best remains to spin on the minimal photoresist thickness required for the next silicon etching step. As the silicon to resist etching rates selectivity is 50:1 with an etching depth of typically $10-20 \mu \mathrm{m}$, the resist thickness required is $0.5 \mu \mathrm{m}$ at the most. We therefore choose as a resist the 1:1 mixture of $\mathrm{AZ} 1512 \mathrm{HS}$ photoresist:AZ EBR solvent. This allows a $0.6 \mu \mathrm{m}$ thick resist onto the silicon samples using $3000 \mathrm{rpm}$ for $30 \mathrm{~s}$ spin on. This resist thickness could be naturally reduced toward $0.3 \mu \mathrm{m}$ for $10 \mu \mathrm{m}$ etching using a larger AZ EBR proportion in the AZ 1512 HS photoresist:AZ EBR solvent mixture.

Once the resist is developed [Fig. 3(b)], the samples are etched in the STS cluster. The most critical parameter seems to be the oxygen flow in the etching gas mixture, which makes the deposition layer removal at the bottom of the etched features easier. However, a too high oxygen flow level may partially oxidize the etched silicon surface, leading to the formation of "black silicon" (tiny nanowires of silicon).

The etched features are then filled up with liquid PDMS mixture using a 10:1 base to curing agent ratio in mass. At this step, Fig. 3(d) shows that the PDMS is planarized before thermal cross-linking. Several attempts have been undertaken to achieve the right PDMS planarization that allows removing the cross-linked PDMS residual layer by plasma: (i) The simple sample spinning after mold filling up with PDMS does not produce a flat surface of the PDMS residual layer (roughness of a few micrometers). In addition, the PDMS residual layer thickness cannot be reduced below about $10 \mu \mathrm{m}$. These two drawbacks do not permit performing plasma planarization afterward. (ii) The use of a flat silicon master or polyethylene film (between PDMS and a flat silicon master) coated with a silane antisticking layer (Optool DSX, Daikin, with a surface energy of $10 \mathrm{~mJ} / \mathrm{m}^{2}$ here) during the PDMS thermal cross-linking does not achieve good demolding results. These solutions end up with damaged cross-linked PDMS structures when the silicon master is removed or the film is peeled, despite these antisticking coatings providing very good results in imprint lithography for $200 \mathrm{~mm}$ diameter silicon masters demolding. ${ }^{38}$ We observed that the affinity between cross-linked PDMS and photoresists is smaller than the two formerly mentioned solutions. Optool DSX coating is therefore replaced by an AZ 1512 HS resist coating on the polyethylene film. This choice appears as the key point for successful PDMS planarization. A PDMS residual thickness of less than $1 \mu \mathrm{m}$ is easily achieved by applying a squeezing force of $50 \mathrm{~N}$ for at least 1 min on the silicon master. After PDMS thermal crosslinking, the peeling of the polyethylene film does not cause any damage to the PDMS structures. The cross-linked PDMS layer at the upper surface is removed by dry etching it in the previously described DPS etching reactor [Fig. 3(f)]. As the cross-linked PDMS etch rate is close to $1 \mu \mathrm{m} / \mathrm{min}$ using a 2:1 $\mathrm{CF}_{4}: \mathrm{O}_{2}$ gas mixture (see Sec. II), the opening of the top of the hexagonal structures is obtained in $1 \mathrm{~min}$. At the end, the silicon mold could be selectively etched. Aligning a specific lithographic process onto the mold for the selective etching of either the inner part or the outer part of the hexagonal structures is possible. Thus, the opened part may be functionalized. In the following, we indeed etch the entire silicon mold and hide selective regions with a resist. Our constraint is to keep proteins in functional conformations that could be damaged by vacuum conditions. 


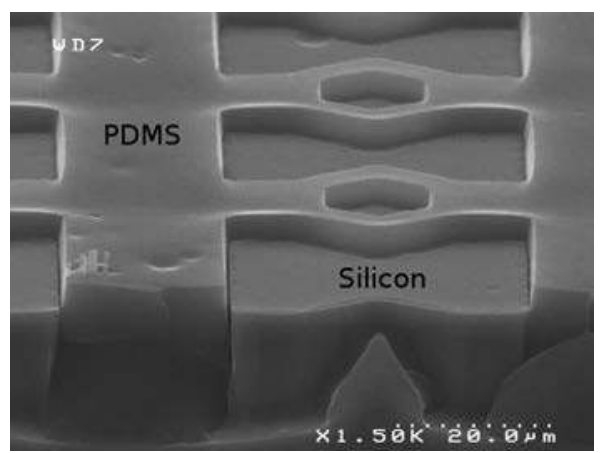

FIG. 4. PDMS frame is removed from the mold by selectively etching the silicon mold.

\section{3D functionalization of the sensor}

The force sensor must host one cell per hexagon and no cells in the rest of the sensor. Thus, internal and external walls of the hexagons must be coated, respectively, with an adhesive and a repulsive coating. As a first step, we choose to coat the adhesive regions with fibronectin. This protein triggers cell/matrix adhesion. For our final purpose, we will need to change fibronectin for some cadherin chimera that contains the extracellular part of the protein targeted to intercellular adhesion, such as $\mathrm{Fc}$ E-cadherin or $\mathrm{Fc}$ $\mathrm{N}$-cadherin. ${ }^{18,39,40}$ Fibronectin is a more easily available protein and we use it to demonstrate the principle of our method. Repulsion of the cells from the desired regions will be ensured by adsorbing Pluronic F127 (Sigma) or bovine serum albumin. ${ }^{41}$ Both preferentially adsorb onto hydrophobic surfaces, while adhesive proteins adsorb onto hydrophilic surfaces.

Standard photolithography steps are performed with an AZ 1512 HS photoresist to hide the regions that must not be coated with the adhesive proteins (see Fig. 5). Oxygen plasma renders the bare surfaces of the PDMS hydrophilic and fibronectin adsorbs onto them. After stripping the resist, the PDMS is hydrophobic, and Pluronic F127 or BSA can adsorb. Pluronic organizes as a brush of polymer ${ }^{42,43}$ that prevents the adsorption of the molecules of the extracellular matrix generated by the cells. ${ }^{41}$ Alignment of the PDMS microstructures with the chromium mask for resist insulation is the crucial step. The precision of the alignment must be below $2 \mu \mathrm{m}$, since the walls of the hexagons are 2 or $3 \mu \mathrm{m}$ wide. However, due to the depths of the PDMS microstructures, the photoresist does not spread as a flat surface, and the sample cannot be approached very close to the mask. Performing good alignment as presented in Fig. 6 can therefore be tedious.

\section{DISCUSSION}

In the present work, we propose an original process that leads to the fabrication of a $10 \mu \mathrm{m}$ thick layer of open structures of PDMS, with 3D local patterning of proteins. Complex 3D patterning strategies have been done before, using hard materials such as resist. ${ }^{27,28}$ However, these strategies do not meet our requirements for the use of deformable bio-
A)

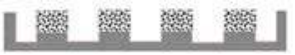

B)

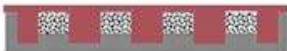

C)

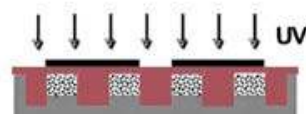

D)

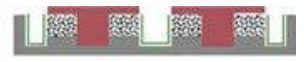

E)

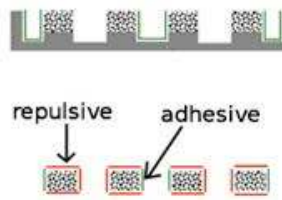

FIG. 5. (Color online) Strategy of surface functionalization. (A) The PDMS frame is still attached to the silicon wafer. (B) A positive photoresist is spun onto the PDMS frame. (C) The regions that are to be treated with adhesive proteins are exposed to UV and developed. (D) After oxidation of the PDMS surface, adhesive proteins are deposited on the surface. (E) The resist is diluted with ethanol and hydrophobic bare surfaces of the PDMS are unveiled. (F) Antiadhesive Pluronic F127 deposits onto the bare surfaces.

compatible materials such as PDMS. The use of photosensitive PDMS could be a way of creating these structures. Unfortunately, the minimum size resolution of the photosensitive PDMS is $10 \mu \mathrm{m}$ in width in the absence of a mold, with a poor pattern anisotropy, ${ }^{36,37}$ while our requirements are $3 \mu \mathrm{m}$ wide and 10-20 $\mu \mathrm{m}$ high anisotropic hexagonal structures. Thus, the design of a mold seems to represent a straightforward way for PDMS hexagonal structure fabrication. The PDMS planarization-for PDMS residual layer reduction above the mold in order to open the other hexagonal structure side-and the PDMS demolding represent two new issues. We present here several simple technological steps to overcome these difficulties by the use of an original PDMS planarization process and the use of the silicon mold as a sacrificial mold.

Although not presented here, the aspect ratio was varied from 3 to 8 (PDMS frame of $20 \mu \mathrm{m}$ high and $2.5 \mu \mathrm{m}$ thick) without observing any additional difficulty. One usual difficulty of making such object is the deformation or the destruction of the frame that occurs when removing the mold. Several authors propose to drive this step into a liquid such as ethanol ${ }^{19}$ to prevent the collapse of the structures. Drying the sample afterward for further selective functionalization steps remains delicate because the capillary forces during evaporation induce the collapse of the structures. The method we propose here circumvents this difficulty of removing the PDMS frame from the mold by selectively etching the silicon mold (Fig. 4). This method requires planarizing and removing the micrometer thick excess of PDMS on the surface of the mold, as detailed in Sec. III B. After etching, the PDMS layer remains bound to the silicon only by its basis, which makes it easy to detach even in dry conditions. Here, we take benefit from the attachment of the PDMS sample to the silicon to perform the functionalization step. The sample is only detached for final use, which minimizes the deformations of the frame.

In the present article, we propose a strategy for 3D functionalization that begins with the adsorption of the adhesive proteins on selected regions and ends up with the deposition of a repulsive coat. This choice is based on the fact that the adhesive proteins we use adsorb onto hydrophilic surfaces. 


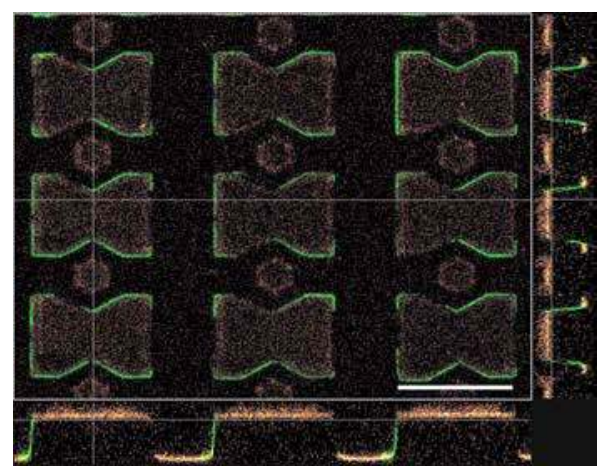

FIG. 6. (Color online) Confocal view of profiles of the PDMS structure. Superposition of the reflection and fluorescent channels. Adhesive proteins appear as thin grey (green color online). In this picture, the inside of the "butterflies" is functionalized with the adhesive proteins. Functionalizing the inside of the hexagons does not change the process except the patterns on the mask. Scale bar $=40 \mu \mathrm{m}$.

In dry conditions, oxidized PDMS surfaces do not remain hydrophilic for more than $0.5 \mathrm{~h}$. Nonreticulated chains migrate back to the surface and restore the hydrophobicity. ${ }^{35} \mathrm{An}$ additional oxidation step would therefore be needed after the adsorption of the repulsive coating. This requires that the repulsive coat is protected from the plasma treatment. Such strategy seems less straightforward than the one we described in Fig. 5. However, the process we developed requires that the adhesive proteins are still efficient after facing ethanol. This is the case for fibronectin, laminin, or collagen, for instance. ${ }^{44}$ A representative view of the substrate after the functionalization step is shown in Fig. 6. Figure 6 shows that proteins successfully adsorb from the top to the bottom of the walls. However, staining is not perfect on the whole perimeter of the wells. This originates from a slight misalignment of the chromium mask with the PDMS frame at step C of the process (see Fig. 5). Such misalignment can be solved with overexposing the resist to enlarge the resist patterns or with working with a mask that contains patterns with size slightly exceeding that of the PDMS patterns.

\section{CONCLUSION}

We described the geometrical and chemical designs and the fabrication of a force sensor designated to measure cellular forces. We proposed a process to make elastomeric, deformable, open structures with a large aspect ratio. The fabrication of a silicon mold designated to PDMS hexagonal structures combines several simple microlithographic processes without significant critical issues. Two original processes are proposed to obtain undeformed PDMS frames: (i) The PDMS planarization process, which is achieved thanks to the use of a flexible polyethylene sheet with an antisticking resist layer spun on that allows easy peeling off without PDMS structures being damaged and easy removal of the PDMS residual layer that is less than $1 \mu \mathrm{m}$ thick. (ii) The detachment of the PDMS from the silicon mold that is done by etching the silicon. Finally, we describe an original strategy to locally functionalize the 3D frame with biological adhesion proteins by hiding selected regions with a resist.
This process can be used for any protein that keeps a functional conformation when dipped into an ethanol bath.

${ }^{1}$ N. Wang, J. P. Butler, and D. E. Ingber, Science 260, 1124 (1993).

${ }^{2}$ Z. Cheng, J. Zhu, P. M. Chaikin, S.-E. Phan, and W. B. Russel, Phys. Rev. E 65, 041405 (2002).

${ }^{3}$ I. B. Bischofs and U. S. Schwarz, Proc. Natl. Acad. Sci. U.S.A. 100, 9274 (2003).

${ }^{4}$ C.-M. Lo, H.-B. Wang, M. Dembo, and Y.-L. Wang, Biophys. J. 79, 144 (2000).

${ }^{5}$ R. J. Pelham and Y. Wang, Proc. Natl. Acad. Sci. U.S.A. 94, 13661 (1997).

${ }^{6}$ D. Choquet, D. P. Felsenfeld, and M. P. Sheetz, Cell 88, 39 (1997).

${ }^{7}$ C. G. Galbraith and M. P. Sheetz, Curr. Opin. Cell Biol. 10, 566 (1998).

${ }^{8}$ B. Geiger and A. D. Bershadsky, Cell 110, 139 (2002).

${ }^{9}$ N. Q. Balaban et al., Nat. Cell Biol. 3, 466 (2001).

${ }^{10}$ J. L. Tan, J. Tien, D. M. Pirone, D. S. Gray, K. Bhadriraju, and C. S. Chen, Proc. Natl. Acad. Sci. U.S.A. 100, 1484 (2003).

${ }^{11}$ A. Saez, A. Buguin, P. Silberzan, and B. Ladoux, Biophys. J. 89, L52 (2005).

${ }^{12}$ C. F. Deroanne, C. M. Lapiere, and B. V. Nusgens, Cardiovasc. Res. 49, 647 (2001).

${ }^{13}$ C. Jamora and E. Fuchs, Nat. Cell Biol. 4, E101 (2002).

${ }^{14}$ L. Tranqui and P. Tracqui, C. R. Acad. Sci. III 323, 31 (2000).

${ }^{15}$ O. du Roure, A. Saez, A. Buguin, R. H. Austin, P. Chavrier, P. Silberzan, and B. Ladoux, Proc. Natl. Acad. Sci. U.S.A. 102, 2390 (2005).

${ }^{16}$ A. Rabodzey, P. Alcaide, F. W. Luscinskas, and B. Ladoux, Biophys. J. 95, 1428 (2008).

${ }^{17}$ Z. Liu, J. L. Tan, D. M. Cohen, M. T. Yang, N. J. Sniadecki, S. A. Ruiz, C. M. Nelson, and C. S. Chen, Proc. Natl. Acad. Sci. U.S.A. 107, 9944 (2010).

${ }^{18}$ A. Ganz, M. Lambert, A. Saez, P. Silberzan, A. Buguin, R. M. Mège, and B. Ladoux, Biol. Cell 98, 721 (2006).

${ }^{19}$ B. Ladoux, E. Anon, M. Lambert, A. Rabodzey, P. Hersen, A. Buguin, P. Silberzan, and R.-M. Mège, Biophys. J. 98, 534 (2010).

${ }^{20}$ N. J. Sniadecki, A. Anguelouch, M. T. Yang, C. M. Lamb, Z. Liu, S. B. Kirschner, Y. Liu, D. H. Reich, and C. S. Chen, Proc. Natl. Acad. Sci. U.S.A. 104, 14553 (2007).

${ }^{21}$ K. A. Kilian, B. Bugarija, B. T. Lahn, and M. Mrksich, Proc. Natl. Acad. Sci. U.S.A. 107, 4872 (2010).

${ }^{22}$ M. Théry and M. Bornens, Curr. Opin. Cell Biol. 18, 648 (2006).

${ }^{23}$ M. Théry, A. Pépin, E. Dressaire, Y. Chen, and M. Bornens, Cell Motil. Cytoskeleton 63, 341 (2006).

${ }^{24}$ M. Cavey, M. Rauzi, P.-F. Lenne, and T. Lecuit, Nature (London) 453, 751 (2008)

${ }^{25}$ B. Alberts, D. Bray, J. Lewis, M. Raff, K. Roberts, and J. D. Watson, Molecular Biology of the Cell, 3rd ed. (Garland, New York, 1994).

${ }^{26}$ U. Schwarz, Soft Matter 3, 263 (2007).

${ }^{27}$ J. Kettle, R. T. Hoyle, R. M. Perks, and S. Dimov, J. Vac. Sci. Technol. B 26, 1794 (2008).

${ }^{28}$ N. Kehagias et al., Nanotechnology 18, 175303 (2007).

${ }^{29}$ F. Lärmer and A. Schilp, Patent No. DE4241045 (1992); Patent No. US 5501893 (1996); Patent No. EP 625285 (1994).

${ }^{30}$ D. Fuard, T. Tzvetkova-Chevolleau, S. Decossas, P. Tracqui, and P. Schiavone, Microelectron. Eng. 85, 1289 (2008).

${ }^{31}$ S. Ma, M. Jain, and J. D. Chinn, J. Vac. Sci. Technol. A 16, 1440 (1998).

${ }^{32}$ E. Pargon, M. Martin, J. Thiault, O. Joubert, J. Foucher, and T. Lill, J. Vac. Sci. Technol. B 26, 1011 (2008).

${ }^{33}$ J. Garra, T. Long, J. Currie, T. Schneider, R. White, and M. Paranjape, J. Vac. Sci. Technol. A 20, 975 (2002).

${ }^{34}$ S. R. Oh, J. Micromech. Microeng. 18, 115025 (2008).

${ }^{35}$ M. Morra, E. Occhiello, R. Marola, F. Garbassi, P. Humphrey, and D. Johnson, J. Colloid Interface Sci. 137, 11 (1990).

${ }^{36}$ S. P. Desai, B. M. Taff, and J. Voldman, Langmuir 24, 575 (2008).

${ }^{37}$ T. Honegger, K. Berton, T. Pinedo-Rivera, and D. Peyrade, Microelectron. Eng. 86, 1401 (2009).

${ }^{38}$ M. Zelsmann et al., J. Vac. Sci. Technol. B 27, 2873 (2009). 
${ }^{39}$ J. Tsai and L. Kam, Biophys. J. 96, L39 (2009).

${ }^{40}$ I. Dupin, E. Camand, and S. Etienne-Manneville, J. Cell Biol. 185, 779 (2009).

${ }^{41}$ C. M. Nelson, S. Raghavan, J. L. Tan, and C. S. Chen, Langmuir 19, 1493 (2003).
${ }^{42}$ M. R. Nejadnik, A. L. J. Olsson, P. K. Sharma, H. C. van der Mei, W. Norde, and H. J. Busscher, Langmuir 25, 6245 (2009).

${ }^{43}$ X. Liu, D. Wu, S. Turgman-Cohen, J. Genzer, T. W. Theyson, and O. J. Rojas, Langmuir 26, 9565 (2010).

${ }^{44}$ Z. Gatalica and I. Damjanov, Histochemistry 95, 189 (1990). 